\title{
DIFFUSE LARGE B-CELL LYMPHOMA WITH NEUROLYMPHOMATOSIS MIMICKING SPONDYLOARTHRITIS: A DIAGNOSTIC CHALLENGE
}

Júlia Boechat Farani ${ }^{1 \star \star}$, Erika Biegelmeyer ${ }^{1}$, Evelise Berlet ${ }^{1}$, Samanta Gerhardt ${ }^{1}$, Viviane Elisa Timm ${ }^{1}$, Mauro Waldemar Keiserman $^{1}$, Henrique Luiz Staub ${ }^{1}$ 1.Pontifícia Universidade Católica, Porto Alegre (RS), Brazil.

*Corresponding author: juliaboechat@globo.com

\section{BACKGROUND}

Lymphoid neoplasms can mimic a wide range of autoimmune disorders, but rarely spondylarthritis.

\section{CASE REPORT}

A 58-year-old white immunocompetent woman with previous history of smoking and arterial hypertension was admitted to our unit with a two-month history of widespread neuropathic pain, diplopia, bilateral peripheral facial paralysis, dysarthria, dysphagia and abnormal gait. Upon examination, there was tactile allodynia and symmetrical proximal weakness of lower limbs. Magnetic resonance imaging (MRI) initially showed intracranial hypertension and a slight enhancement of the VII cranial nerve. Cerebrospinal fluid (CSF) analysis demonstrated albuminocytological dissociation and microbiological workup was negative. Electroneuromyography revealed a demyelinating sensorimotor polyneuropathy of four limbs. Symptoms progressed despite methylprednisolone pulse therapy and intravenous immunoglobulin (IVlg). During investigation, she developed acute monoarthritis of the right knee and inflammatory back pain. Inflammatory markers were elevated, and synovial fluid was inflammatory, with negative cultures. There were high serum IgG antibodies to Chlamydia spp. Laboratory search was negative for HLA-B27, rheumatoid factor, antinuclear and antineutrophil cytoplasmic antibodies. Magnetic resonance imaging suggested active sacroiliitis with subchondral sclerosis and slight bone marrow edema on the right side. She was treated with doxycycline for 21 days. Sulfasalazine was initiated but suspended due to hemolytic anemia. In subsequent months, the patient experienced progressive tetraparesis. Magnetic resonance imaging was repeated and revealed T2 and FLAIR periventricular hyperintense lesions and myeloradiculopathy, suggesting combined central and peripheral demyelination. Cerebrospinal fluid analysis exhibited increasing protein levels and hypoglycorrhachia with no signs of infection. Serum immunofixation demonstrated oligoclonal bands. Onconeural antibodies were not detected. She was refractory to IVIg and 2 monthly doses of cyclophosphamide. Within one year after initial presentation, she could not walk and had severe uncontrolled neuropathic and inflammatory back pain. Computer tomography (CT) scans showed right iliac bone sclerosis surpassing the subchondral area (Fig. 1). Finally, a new CSF analysis showed pleocytosis with 99\% lymphocytes, and flow cytometry immunophenotyping revealed a clonal population, compatible with neurolymphomatosis by diffuse large B-cell lymphoma (DLBCL). Positron-emission tomography suggested active lymphoproliferative disease in brain lesions, nerve roots, as well as the right iliac bone, sacrum and iliac wing. A recent MRI demonstrated small diffuse pelvic lesions suggestive of lymphoid infiltration (Fig. 2). She developed SARS-Cov-2 pneumonia, so hemato-oncologic treatment was not initiated due to poor performance status.

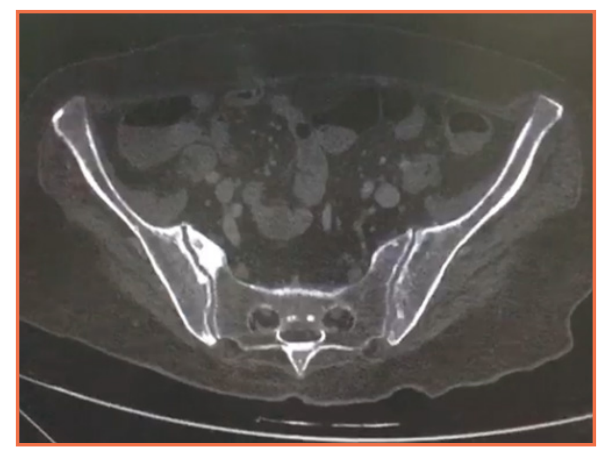

Figure 1. CT scan displaying right iliac bone sclerosis extending to a significant distance from the joint space, surpassing the subchondral area.

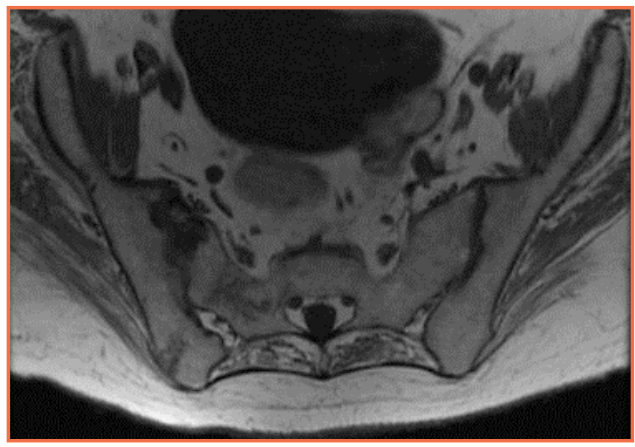

Figure 2. T1-weighted MRI with fat suppression showing diffuse pelvic lesions suggestive of lymphoid infiltration.

\section{CONCLUSION}

This is a rare case of DLBCL with neurolymphomatosis mimicking spondyloarthritis. Atypical radiological findings brought attention to non-classical sacroiliitis, probably due to bone marrow lymphoid infiltration. 